\title{
Diabetomics- A Potential Saliva Based Diabetes Diagnosis
}

\author{
Prerana G ${ }^{1}$, Aishwarya P R Kumar ${ }^{1}$ and Gaurav ${ }^{2 *}$ \\ ${ }^{1}$ House Surgeons, NSVK Sri Venkateshwara Dental College and Hospital, Bangalore, \\ Karnataka, India \\ ${ }^{2}$ Consultant Oral Physician and Maxillofacial Radiologist, Assistant Professor, \\ Department of Oral Medicine and Maxillofacial Radiology, NSVK Sri Venkateshwara \\ Dental College and Hospital, Bangalore, Karnataka, India \\ *Corresponding Author: Gaurav, Consultant Oral Physician and Maxillofacial \\ Radiologist, Assistant Professor, Department of Oral Medicine and Maxillofacial \\ Radiology, NSVK Sri Venkateshwara Dental College and Hospital, Bangalore, \\ Karnataka, India.
}

\section{Abstract}

Background: With 400 million individuals suffering from one of the most prevalent conditions known to mankind diabetes; there still exists an age old invasive diagnostic and self-assessment criteria which is till date considered to be the gold standard. Everyday approximately 0.4 - 2.8 litres of saliva is produced within the oral cavity; which raises a question as to why we cannot use this human serum to diagnose diabetes. The abundance of biomarkers such as genetic material and protein it contains, it is hard to ignore this as a beginning of a new era of using saliva as a potent non-invasive diagnostic tool.

Aim of the Study: To assess the significance of salivary proteins in diagnosis of Type 2 Diabetes (Insulin Independent Diabetes Mellitus).

Research Question: Is Diabetomics actually significant enough in diagnosing Type 2 Diabetes (Insulin Independent Diabetes Mellitus).

Materials and Methods: With the Medline database taken as a source for authenticated scientific research data including few others like Cochrane and Medknow, 55 articles were selected having undergone Randomized Control Trial (RCT). Out of these, 19 articles (studies) were chosen which met the inclusion and exclusion criterion for systematic review.

Results and Conclusion: The conventional methods of diagnosis have a lot of demerits associated with it. Diabetomics on the other hand, can be used as a potential diagnostic tool in the diagnosis of Diabetes Mellitus Type 2. Saliva being a complex fluid with its constituents similar to that of plasma can be unmistakably devoted to the field of diagnosis. Not only can it serve as a diagnostic aid for Diabetes, if the salivary biomarkers are utilized to its full potential, it can be used for the diagnosis of a plethora of diseases [1]. Keywords: Diabetes Mellitus; Diabetomics; Type 2 Diabetes

\section{Introduction}

Diabetes mellitus is simple words that increase in the blood glucose levels of a person over a long period of time. The prevalence of
Diabetes Mellitus is growing rapidly around the world. According to the reports by the Indian Council of Medical Research and Institute of Health Metrics and Evaluation it has been projected that the

Citation: Gaurav., et al. "Diabetomics- A Potential Saliva Based Diabetes Diagnosis". Acta Scientific Dental Sciences 5.6 (2021): $98-103$. 
rate of growth of diabetes type 2 will be $7.2 \%$ in India alone in the next 5 years adding to the already existing 415 million worldwide. But the problem doesn't end here, about $40 \%$ of the people with type 2 diabetes go undiagnosed which is roughly 166 million cases worldwide.

Presently, Diabetes mellitus is diagnosed by evaluating blood sugar levels which is an invasive way of investigation. With growing cases, it is very important to come up with a technique which is non-invasive and is much simpler.

One of the fastest growing areas of research is using salivary biomarkers [2] as a diagnostic parameter to determine the existence or risk of developing diabetes mellitus and to even monitor the response to a particular treatment. Using saliva helps us in noninvasive and frequent sampling of biomarkers. Furthermore, saliva is considered to have similar proteomics with blood and hence is a significant diagnostic tool.

The employment of such a simple and non-invasive technique of a body fluid such as saliva can act as a substantial protocol for diagnosing and managing diabetes at a larger scale both in the local and global arena. This simple diagnostic tool can be a breakthrough for many countries which are devoid of facilities.

\section{Aim of the Study}

To assess the significance of salivary proteins in diagnosis of Type 2 Diabetes (Insulin Independent Diabetes Mellitus).

\section{Research Question}

Is Diabetomics actually significant enough in diagnosing Type 2 Diabetes (Insulin Independent Diabetes Mellitus)

\section{Materials and Methods}

With the Medline database taken as a source for authenticated scientific research data including few others like Cochrane and Medknow, 55 articles were selected having undergone Randomized Control Trial (RCT). Out of these, articles (studies) 19 were chosen which met the inclusion and exclusion criterion for systematic review.

\section{Results}

The field of Diabetomics is very significant in the Diagnosis of Type 2 Diabetes. It possesses a number of advantages over the con- ventional methods. To name a few:
a. Highly sensitive and specific nature
b. Non- invasive.
c. Patient comfort.
d. Economic.

\section{Discussion}

Diabetes Mellitus is a metabolic disease that is caused as a result of defective insulin secretion/function or sometimes both. While there are many types of diabetes, type 2 Diabetes in particular is due to high sugar levels circulating in the body. It is estimated that nearly around 460 million adults are currently living with Diabetes out of which $90 \%$ of cases account for type 2 Diabetes. The Diabetes Mellitus is a disease which is more prevalent in Developed countries. Some of the most common reasons for the increase in incidence of Diabetes include sedentary lifestyles, less physically demanding work, increased food intake etc. According to the WHO, nearly 1.6 billion people die because of Diabetes each year. The symptoms of Diabetes include increased thirst, frequent urination, hunger, fatigue, blurred vision etc. In some cases, there might not be any presenting symptoms, in such cases diagnosing might become challenging.

While there are many ways to Diagnose diabetes, the most commonly used methods are $\mathrm{HbA} 1 \mathrm{C}$ criteria or Plasma glucose concentration. Both are invasive methods in which blood samples are collected from the candidate and assessed for sugar levels. The plasma glucose concentration method is based on fasting plasma glucose levels (Table 1) (in which a blood sample is taken after an 8 hour fast) or oral glucose tolerance test (Table 2) (OGTT - in which a blood sample is taken 2 - 3 hours before and after the meal). The diagnosis is made after assessing the results based on the standard criteria given by American Diabetes Association. A1C measures the average blood sugar levels over the past 2 - 3 months (Table 3).

\begin{tabular}{|c|c|}
\hline Result & Fasting Plasma Glucose \\
\hline Normal & Less than $100 \mathrm{mg} / \mathrm{dl}$ \\
\hline Pre-diabetes & $100 \mathrm{mg} / \mathrm{dl}$ to $125 \mathrm{mg} / \mathrm{dl}$ \\
\hline Diabetes & $126 \mathrm{mg} / \mathrm{dl}$ or higher \\
\hline
\end{tabular}

Table 1 


\begin{tabular}{|c|c|}
\hline Result & Oral Glucose Tolerance Test (OGTT) \\
\hline Normal & Less than $140 \mathrm{mg} / \mathrm{dl}$ \\
\hline Pre-diabetes & $140 \mathrm{mg} / \mathrm{dl}$ to $199 \mathrm{mg} / \mathrm{dl}$ \\
\hline Diabetes & $200 \mathrm{mg} / \mathrm{dl}$ or higher \\
\hline
\end{tabular}

Table 2

\begin{tabular}{|c|c|}
\hline Result & A1C \\
\hline Normal & Less than $5.7 \%$ \\
\hline Pre-diabetes & $5.7 \%-6.4 \%$ \\
\hline Diabetes & $6.5 \%$ or higher \\
\hline
\end{tabular}

Table 3

Demerits of conventional methods of diagnosing diabetes:

1. Invasive technique of sample collection

2. Highly uncomfortable for patients

3. Risk of infection from bruised skin

Significance of saliva in diagnosis

Saliva is a complex biologic fluid that has been used as a diagnostic tool for more than 2000 years. In fact, for many years now Saliva and blood are known to be brothers as they come from the same source. Many changes in the composition of saliva are a result of alterations in the overall metabolism of the body. In the $19^{\text {th }}$ century, researchers had learnt that saliva had digestive effects. But in recent times, it has been discovered that Saliva has much more potential in the field of diagnosis.

The branch of salivaomics basically deals with analysis of Saliva to identify molecules (bio markers) which are specific to many endocrine $[3,4]$, inflammatory and infectious diseases. As the saying goes, the best treatment for any disease involves early detection. Diagnosing the disease can be a great deal. Salivary diagnostics seems to be very promising and reliable for the detection of a plethora of diseases.

Saliva is $99.5 \%$ made of water. The rest $0.5 \%$ is constituted of organic and inorganic constituents out of which, the main lime light is on the protein content. Researchers have now identified a number of proteins that have the potential for diagnosis [5]. Proteomics is a large scale study of proteins. It is a set of proteins produced in an organism, system or biological context.

Salivary biomarkers

By definition, a biomarker is "a characteristic that is objectively measured and evaluated as an indicator of normal biologic process, pathologic process, and pharmacological response to a therapeutic intervention".

There are a range of biomarkers which are used today. A simple example of a biomarker is the rise in the body temperature for fever. According to studies, they are various molecular and microbial by-products. These revelations have formed the foundation field of salivary diagnosis. To name a few Salivary biomarkers - DNA, RNA, Proteins, Mucins, Immunoglobulins, Metabolites, Viruses, Bacteria and other cellular materials.

The possible use of salivary biomarkers:

- Diagnosis of carcinomas.

- Diagnosis of endocrine diseases.

- $\quad$ Monitoring drug use.

- Detecting dental caries.

- $\quad$ Standard genotyping.

- $\quad$ Forensics.

\section{Salivary proteome}

Looking into the origin of salivary proteome, we can see that proteomes are derived from glandular secretions, oral bacterial products or GCF. Few of the families of proteins which can be seen are proline rich, amylase and Mucin [6]. The major contribution to the salivary proteome comes through GCF.

\section{Saliva and diabetes}

When we speak of endocrine disturbances, diabetes has been prevalent for centuries. Many of the diabetes cases go undetected for years. Though diagnosis of diabetes has not been that challenging, there are no established criteria as such which can be relied upon. The current methods of diagnosis include monitoring blood glucose levels or HBA1C for long term. However, they come with 
many demerits. But now the question arises as to how Saliva can be useful in the diagnosis of Diabetes mellitus 2 .

There are 2 methods of establishing the diagnosis of Diabetes mellitus 2 through Saliva [7]:

- Estimation of glycosylation of salivary proteins.

- Identifying the specific biomarkers involved in Diabetes mellitus type 2 .

Mechanism through which glycosylation occurs [7,8]:

1. Glucose links covalently to target proteins through the formation of Schiff base.

2. Schiff base is formed between aldehyde of glucose and amino acid of lysine.

3. Amadori rearrangement and oxidation.

4. Advanced glycation end products.

Mechanism through which specific protein biomarkers are formed:

1. Hyperglycaemia.

2. Increase in glucose flux through hexosamine glycosylation pathway.

3. Provides UDP-GlcNAC and GalNAC precursors for addition of various carbohydrate moieties to proteins.

4. This occurs through beta linked 0-glycosylation of intercellular proteins.

5. Alpha linked mucin-type 0 - and $\beta$-linked N-glycosylation of cell-surface and secreted proteins.

Procedure-Multidimensional liquid chromatography/tandem mass spectroscopy:

- Label free quantification is generally used to identify the proteomes.

- Immunodetection is employed to confirm the potential biomarkers.
Most commonly found diabetes proteomic biomarkers in saliva $[10,11]$

Group A - Metabolites

\begin{tabular}{|c|}
\hline Carbonic anhydrase 6 \\
\hline Phosphoglycerate kinase \\
\hline Carbonic anhydrase 1 \\
\hline Carbonic anhydrase 2 \\
\hline
\end{tabular}

Group B - Immune response

\begin{tabular}{|c|}
\hline Calnexin \\
\hline Protein plunk \\
\hline Alpha 1-antitrypsin \\
\hline Annexin-A \\
\hline
\end{tabular}

Group C - Development

\begin{tabular}{|c|}
\hline Fatty acid binding protein \\
\hline Peroxiredoxin \\
\hline Laminin A/C \\
\hline Desmoplakin \\
\hline
\end{tabular}

The other group of proteins includes Extracellular matrix protein, signal transduction Protein, cell organization and biogenesis, cell motility proteins. However, the existence of these proteins in Diabetes mellitus type 2 is of questionable significance. 487 unique proteins have been identified so far out of which $1 / 3^{\text {rd }}$ of them were previously not reported in human saliva [9].

Another study concluded that the levels of alpha-2 macroglobulin [11] (glycoprotein) are significantly increased in patients with Diabetes compared to the control group. Alpha-2 macroglobulin has been associated with many diseases; it is a glycoprotein which is generated in the liver. The salivary Alpha-2 macroglobulin has been associated with Diabetes mellitus type 2 .

Group A- Metabolites

Carbonic anhydrase 1

Carbonic anhydrase 1 belongs to the family of zinc metalloenzyme. They are present in CSF, saliva and gastric acid. Elevated 
levels of CA1 have been associated with ischemic cardiomyopathy, diabetes mellitus type 2 . Also, glucose induced elevation of CA1 hampers endothelial cell permeability.

\section{Carbonic anhydrase 2}

CA2 is an enzyme whose main function is to catalyse the reversible hydration of carbon dioxide. Elevated levels of this enzyme are significantly increased in type 2 Diabetes according to research. It has also been associated with osteoporosis, renal tubular acidosis etc.

\section{Carbonic anhydrase 6}

CA6 is abundantly found in saliva. It is also called Gustin. The major function of CA6 in saliva is not understood. However elevated levels of CA6 were found in Diabetes mellitus type 2 according to studies. CA6 has been suggested to participate in the maintenance of homeostasis in oral and GI environments.

\section{Phosphoglycerate kinase}

Phosphoglycerate kinase is an enzyme involved in glycolysis. Though the levels of this enzyme have been said to be increased in Diabetes mellitus type 2, its main significance was found to be in Diabetes mellitus type 1 . The PGK activity was significantly ( $P$ less than 0.001) elevated in diabetes, however it is restored to normalcy after insulin treatment.

\section{Group B- Immune response}

Calnexin

Calnexin is a chaperone; it assists in the protein folding and quality control. It plays an important role by ensuring that only properly folded proteins take part in the further processes.

\section{Protein Plunc}

Palate, lung and nasal epithelium clone protein (PLUNC) is a gene encoding a secretory protein which is seen in the upper respiratory tract. Plunc is said to have antimicrobial functions and has a role in innate immune defence. The levels of this protein were significantly high in Diabetes mellitus type 2 according to studies.

\section{Alpha-1 antitrypsin}

Many studies have shown that the decreased levels of Alpha-1 antitrypsin are associated with both Diabetes mellitus type 1 and
2. According to a research, the number of individuals with low AAT levels was $50 \%$ higher in the group with diabetes and the frequency of AAT deficiency genotypes was 50\% higher in diabetic patients.

\section{Annexin A}

Annexin group of proteins is a very common group of proteins. They are associated with anti-inflammatory action, coagulation and fibrinolysis. According to research, the levels of Annexin-A was positively associated with people with Obesity and type 2 Diabetes.

\section{Group C- Development}

Fatty acid binding protein

Fatty acid binding proteins are a family of transport proteins associated with lipophilic substances. The FABP plays a role in glucose homeostasis. The levels of FABP were abundantly expressed which confers an increased risk of type 2 Diabetes according to American Diabetes Association.

\section{Peroxiredoxin}

Peroxiredoxin are the family of antioxidant enzymes and also mediate signal transduction. Increased oxidative stress alters the metabolism. It is found that peroxiredoxin is a mediator of overt hyperglycaemia in type 2 Diabetes.

\section{Laminin A/C}

Laminins as commonly known are the key components of the basal lamina. The gene encoding for Laminin protein is LMNA gene. The expression of this gene is altered in obesity and type 2 Diabetes mellitus. The levels of Laminin A and C mRNA were found to be increased in obese and diabetic patients according to a research.

\section{Desmoplakin}

Desmoplakin is the protein encoded by the DSP gene. It is a critical component of desmosome structures. The exact association between Desmoplakin and Diabetes is not very well understood. However studies have found the association of Desmoplakin with insulin signalling.

\section{Advantages of using saliva as a diagnosing tool:}

1. Painless and non-invasive.

2. Sample collection is easy. 
3. Economic.

4. Can be safely carried out in children.

Disadvantages of using saliva as a diagnosing tool:

1. Requires high sensitivity and specificity.

2. May not be suitable for other types of diabetes.

The results from various studies are suggesting that salivary proteomic biomarkers have a tremendous capacity to be a diagnostic tool. In the near future, it is expected that a simple spit from the patient would be enough for the screening of a variety of disease conditions. There are a number of these precious moieties which are yet to be discovered [12].

\section{Conclusion}

The conventional methods of diagnosis have a lot of demerits associated with it. Diabetomics on the other hand, can be used as a potential diagnostic tool in the diagnosis of Diabetes Mellitus Type 2. Saliva being a complex fluid with its constituents similar to that of plasma can be unmistakably devoted to the field of diagnosis. Not only can it serve as a diagnostic aid for Diabetes, if the salivary biomarkers are utilized to its full potential, it can be used for the diagnosis of a plethora of diseases.

\section{Bibliography}

1. Agho ET., et al. "Salivary inflammatory biomarkers and glycated haemoglobin among patients with type 2 diabetic mellitus". BMC Oral Health 21 (2021): 101.

2. Ladgotra Amit., et al. "Estimation of Salivary and Serum Biomarkers in Diabetic and Non Diabetic Patients - A Comparative Study". Journal of Clinical and Diagnostic Research: JCDR 10.6 (2016): ZC56-61.

3. Pérez-Ros Pilar, et al. "Changes in Salivary Amylase and Glucose in Diabetes: A Scoping Review”. Diagnostics 11.3 (2021): 453.

4. Yoshizawa Janice M., et al. "Salivary biomarkers: toward future clinical and diagnostic utilities". Clinical Microbiology Reviews 26.4 (2013): 781-791.
5. Rastogi V., et al. "Relationship between Salivary Alpha-2 Macroglobulin and HbA1c among Patients with Type-2 Diabetes Mellitus: A Cross-sectional Study". Indian Journal of Endocrinology and Metabolism 23 (2019): 184-187.

6. Khera S and Saigal A. "Assessment and evaluation of gustatory functions in patients with diabetes mellitus Type II: A study". Indian Journal of Endocrinology and Metabolism 22 (2018): 204-207.

7. Srinivasan Mythily., et al. "Literature-based discovery of salivary biomarkers for type 2 diabetes mellitus". Biomarker Insights 10 (2015): 39-45.

8. Agho Ekhosuehi Theophilus., et al. "Salivary inflammatory biomarkers and glycated haemoglobin among patients with type 2 diabetic mellitus". BMC Oral Health 21.1 (2021): 101.

9. Caixeta Douglas C., et al. "Salivary molecular spectroscopy: A sustainable, rapid and non-invasive monitoring tool for diabetes mellitus during insulin treatment". PloS one 15.3 (2020): e0223461.

10. Guo Lin-Na., et al. "Serum and salivary ferritin and Hepcidin levels in patients with chronic periodontitis and type 2 diabetes mellitus". BMC Oral Health 18.1 (2018): 63.

11. Mythily Srinivasan., et al. "Assessment of Salivary Adipokines Resistin, Visfatin, and Ghrelin as Type 2 Diabetes Mellitus Biomarkers". Biochemistry Research International (2018).

12. Desai P., et al. "The Clinical Utility of Salivary Biomarkers in the Identification of Type 2 Diabetes Risk and Metabolic Syndrome". Diabetes, Metabolic Syndrome and Obesity: Targets and Therapy 13 (2020): 3587-3599.

\section{Volume 5 Issue 6 June 2021 (C) All rights are reserved by Gaurav.., et al.}

